\title{
Light-dependent expression of flg22-induced defense genes in Arabidopsis
}

\author{
Satoshi Sano ${ }^{1+}$, Mayu Aoyama ${ }^{1+}$, Kana Nakai ${ }^{1}$, Koji Shimotani $^{1}$, Kanako Yamasaki ${ }^{1}$, Masa H. Sato ${ }^{1}$, \\ Daisuke Tojo ${ }^{1}$, I. Nengah Suwastika ${ }^{2}$, Hironari Nomura ${ }^{3}$ and Takashi Shiina ${ }^{1 *}$ \\ Graduate School of Life and Environmental Sciences, Kyoto Prefectural University, Kyoto, Japan \\ 2 Biology Department, Faculty of Science, Tadulako University, Palu, Indonesia \\ ${ }^{3}$ Department of Health and Nutrition, Gifu Women's University, Gifu, Japan
}

Edited by:

Cris Argueso, Colorado State

University, USA

Reviewed by:

Karin Krupinska, Christian-Albrechts

University of Kiel, Germany

Saijaliisa Kangasjärvi, University of

Turku, Finland

Mitsumasa Hanaoka, Chiba

University, Japan

\section{*Correspondence:}

Takashi Shiina, Graduate School of Life and Environmental Sciences,

Kyoto Prefectural University,

Shimogamo, Sakyo-ku,

Kyoto 606-8522, Japan

e-mail: shiina@kpu.ac.jp

these authors have contributed equally to this work.
Chloroplasts have been reported to generate retrograde immune signals that activate defense gene expression in the nucleus. However, the roles of light and photosynthesis in plant immunity remain largely elusive. In this study, we evaluated the effects of light on the expression of defense genes induced by flg22, a peptide derived from bacterial flagellins which acts as a potent elicitor in plants. Whole-transcriptome analysis of flg22-treated Arabidopsis thaliana seedlings under light and dark conditions for 30 min revealed that a number of $(30 \%)$ genes strongly induced by flg22 (>4.0) require light for their rapid expression, whereas flg22-repressed genes include a significant number of genes that are down-regulated by light. Furthermore, light is responsible for the flg22-induced accumulation of salicylic acid (SA), indicating that light is indispensable for basal defense responses in plants. To elucidate the role of photosynthesis in defense, we further examined flg22-induced defense gene expression in the presence of specific inhibitors of photosynthetic electron transport: 3-(3,4-dichlorophenyl)-1,1-dimethylurea (DCMU) and 2,5-dibromo-3-methyl-6-isopropyl-benzoquinone (DBMIB). Light-dependent expression of defense genes was largely suppressed by DBMIB, but only partially suppressed by DCMU. These findings suggest that photosynthetic electron flow plays a role in controlling the light-dependent expression of flg22-inducible defense genes.

Keywords: photosynthesis, flg22, defense gene, DBMIB, DCMU, retrograde signaling, salicylic acid, CAS

\section{INTRODUCTION}

Over the course of their evolution, plants have developed defense systems against a broad-spectrum of pathogens. Plant cells recognize pathogens through pattern-recognition receptors (PRRs) that recognize common features of microbial pathogens, termed pathogen-associated molecular patterns (PAMPs). The recognition of PAMPs by PRRs rapidly initiates downstream signaling events that result in the activation of an array of basal defense responses (PAMP-triggered immunity, PTI; Chisholm et al., 2006; Göhre and Robatzek, 2008). Furthermore, effectortriggered immunity (ETI) induces cell death at infection sites to enclose the spread of pathogens, a process also known as the hypersensitive reaction (HR). Plant immunity activates signal transduction pathways such as the mitogen-activated protein kinase (MAPK) phosphorylation cascades, and $\mathrm{Ca}^{2+}$ and reactive oxygen species (ROS) signaling pathways, which lead to transcriptional reprogramming and defense responses, including the accumulation of salicylic acid (SA), a critical signaling molecule in plant immunity. There are two distinct pathways that produce SA from chorismate in plants: the isochorismate (ICS) pathway in chloroplasts and the phenylalanine ammonia-lyase (PAL) pathway in the cytoplasm. Recently, it was demonstrated that SA is synthesized in chloroplasts via the ICS pathway, but not in the cytoplasm, in Arabidopsis (Fragnière et al., 2011).
PAMPs induce the expression of a specific set of defense genes, a process that is mediated by transcription factors (TFs) such as WRKYs (Rushton et al., 2010; Ishihama et al., 2011). A subset of genes activated by PAMPs is also induced by abiotic stresses such as temperature and drought. Furthermore, plant immune responses are modulated by circadian rhythms as well as abiotic stresses, including light and temperature (Hua, 2013). These facts suggest the presence of crosstalk between biotic and abiotic stress signaling pathways (Fujita et al., 2006).

Light is a fundamental factor in the control of many important biological processes during plant development and environmental responses. There is increasing evidence that light is also required for the appropriate induction of plant defense responses against pathogens (Roberts and Paul, 2006; Kangasjärvi et al., 2012). Zeier et al. (2004) demonstrated that light is responsible for accumulating SA and suppressing bacterial growth. Furthermore, several studies have shown that specific photoreceptors are involved in the regulation of plant immune responses (Griebel and Zeier, 2008; Jeong et al., 2010; Wu and Yang, 2010; Cerrudo et al., 2012). Chloroplasts may also be involved in the light-mediated control of plant immune responses. Göhre et al. (2012) reported that the flg22 peptide derived from bacterial flagellins induces down-regulation of the non-photochemical quenching of excess excitation energy (NPQ) in chloroplasts, 
suggesting a role for chloroplasts in plant immunity. In fact, it was recently demonstrated that the perception of PAMPs generates a transient $\mathrm{Ca}^{2+}$ increase in the chloroplast stroma within a few minuetes (Manzoor et al., 2012; Nomura et al., 2012). These findings suggest that PAMP signals are rapidly relayed to chloroplasts in the early stage of a plant's immune response, and support the idea that chloroplasts mediate light-dependent defense responses against infection by pathogens (Nomura et al., 2012).

Light is not only the energy source for carbon assimilation in chloroplasts, but also an important regulatory factor for chloroplast functions, such as carbon metabolism and other metabolic processes, as well as the expression of chloroplast-encoded genes. In chloroplasts, ROS are unavoidably generated with photosynthetic electron flow, which is driven by light. Singlet oxygen $\left({ }^{1} \mathrm{O}_{2}\right)$ is generated around photosystem II (PS II), and the superoxide anion radical $\left(\mathrm{O}_{2}^{-}\right)$and hydrogen peroxide $\left(\mathrm{H}_{2} \mathrm{O}_{2}\right)$ are generated around photosystem I (PS I). The ${ }^{1} \mathrm{O}_{2}$ and $\mathrm{H}_{2} \mathrm{O}_{2}$ that are photoproduced in the chloroplast mediate retrograde signals to regulate the expression of nuclear-encoded defense genes (Kim et al., 2012; Kangasjärvi et al., 2013; Karpiński et al., 2013; Szechyńska-Hebda and Karpiński, 2013 and the hypersensitive response (Jelenska et al., 2007). CAS has been identified as a thylakoid membranelocalized $\mathrm{Ca}^{2+}$-binding protein that regulates cytoplasmic $\mathrm{Ca}^{2+}$ signals and stomatal closure (Han et al., 2003; Nomura et al., 2008; Vainonen et al., 2008; Weinl et al., 2008). We previously reported that CAS may play a role in the ${ }^{1} \mathrm{O}_{2}$-mediated retrograde signaling for defense responses (Nomura et al., 2012). Based on our findings, we inferred that CAS is involved in the flg22-induced $\mathrm{Ca}^{2+}$ elevation in chloroplasts and in retrograde signaling from the chloroplast to nucleus to control the expression of nuclear-encoded defense genes, including SA biosynthesis genes. Excess light has been shown to activate defense-related genes, possibly through redox changes of the plastoquinone (PQ) pool (Mühlenbock et al., 2008). Furthermore, it has been suggested that the photosynthetic electron transport chain is involved in plant immune (Mateo et al., 2006; Mühlenbock et al., 2008) and stress (Jung et al., 2013) responses. However, the exact role of photosynthesis in the regulation of plant immunity remains unknown.

A large proportion of the biochemical reactions and molecular regulations occurring in chloroplasts is influenced by light. Thus, we predicted that flg22-induced defense gene expression may also be light-dependent. To elucidate the role of light and photosynthesis in flg22-induced defense gene expression, we examined the effects of light/dark conditions and photosynthesis inhibitors on the flg22-regulated expression of nuclear-encoded defense genes. We found that photosynthetic electron flow plays a key role in controlling the light-dependent expression of flg22-inducible defense genes.

\section{MATERIALS AND METHODS PLANT MATERIALS AND GROWTH CONDITIONS}

Arabidopsis thaliana wild-type (WT) Columbia ecotype was used in this study. Sterilized Arabidopsis seeds were germinated on solid agar medium consisting of $0.8 \%(\mathrm{w} / \mathrm{v})$ plant tissue culture grade agar supplemented with $0.5 \times$ Murashige and Skoog
(MS) medium (Wako Chem. Co., Osaka, Japan) and grown at $22^{\circ} \mathrm{C}$ with $16 \mathrm{~h}$ light $\left(80-100 \mu \mathrm{mol} \mathrm{m}{ }^{-2} \mathrm{~s}^{-1}\right) / 8 \mathrm{~h}$ dark cycles for 2 weeks. To avoid mechanical stress when plants were treated with flg22, 2-weeks-old WT plants were floated on $0.5 \times$ strength MS medium for $24 \mathrm{~h}$ before flg2 2 treatment. The dark plants were pre-incubated in the dark for $24 \mathrm{~h}$, while the light plants were illuminated for $4 \mathrm{~h}$ before flg2 2 treatment. Both plants were treated with $1 \mu \mathrm{M}$ flg 22 for $30 \mathrm{~min}$ in the dark or light $(80-100 \mu \mathrm{mol}$ $\left.\mathrm{m}^{-2} \mathrm{~s}^{-1}\right)$. For treatment with photosynthesis inhibitors, plants were incubated with $5 \mu \mathrm{M}$ 2,5-dibromo-3-methyl-6-isopropylbenzoquinone (DBMIB) or $8 \mu \mathrm{M}$ 3-(3,4-dichlorophenyl)-1,1dimethylurea (DCMU) for $30 \mathrm{~min}$ prior to flg22 treatment $(1 \mu \mathrm{M})$. The flg22 peptide was purchased from BIOLOGICA Co. (Nagoya, Japan). DBMIB and DCMU were purchased from Wako Chem. Co. (Osaka, Japan).

\section{MICROARRAY EXPERIMENTS}

The genome-wide microarray analyses were performed using the Arabidopsis V4 2 color microarray (Agilent Technologies). Total RNA was isolated from plants treated with flg22 in the light or dark for 30 min using the Qiagen RNeasy Plant Mini kit following the manufacturer's instructions. Each 200-ng total RNA sample was used to prepare Cy3- or Cy5-labeled target cRNA with the Low Input Quick Amp Labeling Kit (Agilent Technologies, USA) and used in dual color microarray hybridization with the Agilent Arabidopsis v4 oligo microarray slide. A dye-swap experiment was performed with two different RNA populations to eliminate the signal variation caused by the differential labeling efficiency of Cy3 and Cy5 dyes using the SuperScan microarray scanner (Agilent Technologies, USA). The microarray data were normalized by the LOWESS method using Feature Extraction software v. 10.7 (Agilent Technologies) and the expression ratios were analyzed (Non-Uniformity Outlier and Feature Population Outlier). Data with a $P$-value of $>0.01$ were eliminated. The genes showing a consistent expression pattern in the light or dark ( $>2.0$ difference) are listed in sData 1.

\section{MICROARRAY DATA ANALYSIS}

The genes induced by flg22 for $30 \mathrm{~min}$ (Lyons et al., 2013; http://www.nature.com/srep/2013/131009/srep02866/full/srep028 66.html\#supplementary-information) and by illumination of the flu mutant (Laloi et al., 2007) were obtained from the indicated publications. Light-responsive genes in the absence of flg22 were obtained from a public database (Michael et al., 2008; http://www.ebi.ac.uk/arrayexpress/ experiments/E-MEXP-1304/). Gene ontology and MapMan analysis were performed with the Arabidopsis Classification SuperViewer at the BAR of the University of Toronto (http://bar.utoronto.ca/ntools/cgi-bin/ntools_classification_super viewer.cgi). We compared the gene expression profiles from our microarray experiments with available expression data via the expression browser at the BAR. We also searched for overrepresented cis-elements in the 500-bp upstream regions of the downand up-regulated genes in flg22-treated cas-1 plants using the Regulatory Sequence Analysis tool (RSAT; http://rsat.ulb.ac.be/ rsat/). 


\section{qRT-PCR EXPERIMENTS}

Plants were grown and treated with flg22 and photosynthesis inhibitors as described above in Section Plant Materials and Growth Conditions. RNA was extracted from the leaves using the RNeasy Plant Mini kit (Qiagen, USA) and cDNA was generated using SuperScript III (Invitrogen, USA). The Ct values were determined using an iCycler (Bio-Rad, USA) and analyzed with CFX Manager (Bio-Rad, USA). Primers used for qRT-PCR analyses are listed in sTable 1. Expression levels of UBQ10 were constant under flg22 treatments. At least three independent biological replicates were performed for each sample and control. Representative results are shown as the mean \pm s.e.m. of at least three technical experiments.

\section{SA ANALYSIS BY LC/MS}

SA was measured using a conventional high-performance liquid chromatography system. A total of $200 \mathrm{mg}$ of seedling samples without roots was homogenized and extracted with $100 \%$ methanol containing the internal standard anisic acid. Free and glycosylated SA were separated and analyzed by liquid chromatography-tandem mass spectrometry (LC/MS/MS) (3200QTRAP, AB SCIEX, USA) with a modification of the methods described in Nomura et al. (2012).

\section{RESULTS LIGHT-DEPENDENT EXPRESSION OF flg22-INDUCED DEFENSE GENES}

To identify genes rapidly responsive to flg22 whose expression is controlled by light, we performed microarray analysis of lightand dark-dependent gene expression in Arabidopsis seedlings treated with flg22 for $30 \mathrm{~min}$. In flg22-treated plants, expressions of 3192 and 2860 genes significantly increased and decreased (more than two-fold), respectively, in the light compared to the dark control (sData 1). Under our experimental conditions, plants were illuminated for $4 \mathrm{~h}$ before flg22 treatment, whereas dark control plants were kept in the dark. Thus, in order to exclude light-responsive gene sets that are not regulated by flg22, we identified genes that are induced (1612 genes, $>2.0)$ or repressed ( 1496 genes, <0.5) by $4 \mathrm{~h}$ illumination in the absence of flg22 from the public database (Michael et al., 2008). As a result, 616 of 1612 light-induced genes and 699 of 1496 light-repressed genes overlap with the light-dependent and -repressed genes identified in the flg22-treated plants based on our microarray data, respectively. Thus, we removed these genes from our microarray data, and obtained 2576 light-dependent genes and 2161 light-repressed genes in plants treated with flg22 for $30 \mathrm{~min}$. The resultant datasets were used for further analyses.

In order to focus on genes that are rapidly regulated by flg22 in a light-dependent manner, we compared the light-dependent and -repressed genes described above with genes rapidly responsive to flg22 that were identified by Lyons et al. (2013). They showed that flg22 induced the expression of 3579 genes within $30 \mathrm{~min}$ ( $>2.0$ ), whereas it repressed the expression of 4159 genes $(<0.5)$ (Lyons et al., 2013). We identified a large number of genes that are induced by flg22 in a light-dependent manner (sData 2); 536 (14.9\%) of 3579 flg22-induced genes overlapped significantly with the light-dependent genes (named flg22-induced lightdependent genes), but less with light-repressed genes [239 (6.7\%) genes, named flg22-induced light-repressed genes] (Table 1). We further named the genes that are induced by flg22 but lightinsensitive as flg22-induced light-independent genes. As shown in Table 2, the top 25 flg22-induced genes include a large number of light-dependent genes, but not flg22-repressed genes. These results suggest that light is a critical signal for the activation of defense gene expression. Gene ontology analysis revealed that stress-responsive and signal transduction-related genes were overrepresented among the flg22-induced light-dependent genes, but not in the flg22-induced light-repressed genes (sFigure 1). Furthermore, MapMan analysis revealed that functional categories related to stress, signaling, hormone metabolism, and tetrapyrrole synthesis were significantly enriched in the flg22induced light-dependent genes (sTable 2). It should be noted that the light-induced genes include a number of genes involved in SA biosynthesis, such as EDS1, PAD4, SAG101, EDS5, and $P A L 1$, but include fewer TF-related genes compared with the light-independent genes.

Among the 4159 genes suppressed by flg22 within $30 \mathrm{~min}$, 359 were dependent on light (named flg22-repressed lightdependent genes) and 370 genes were repressed by light (named flg22-repressed light-repressed genes). The genes repressed by flg22 and light-insensitive are named flg22-repressed lightindependent genes. The genes rapidly suppressed by flg22 include significantly fewer genes involved in stress responses irrespective of light treatment (sFigure 1). Interestingly, the flg22-repressed light-dependent genes include a large number of genes involved

Table 1 | The number of genes up- and down-regulated by flg22 and light.

\begin{tabular}{|c|c|c|c|c|c|c|}
\hline $\begin{array}{l}\text { Flg22-induced } \\
\text { light-dependent genes }\end{array}$ & \multicolumn{2}{|c|}{ Flg22-responsive genes* } & \multicolumn{2}{|c|}{ Light-responsive genes** } & $\begin{array}{c}\text { Overlap genes } \\
536\end{array}$ & $\begin{array}{r}\boldsymbol{P} \text {-value } \\
8.790 \mathrm{e}-24\end{array}$ \\
\hline $\begin{array}{l}\text { Flg22-repressed } \\
\text { light-dependent genes }\end{array}$ & 4159 & (flg22-down) & 2576 & (light-dependent) & 359 & $2.599 \mathrm{e}-04$ \\
\hline
\end{tabular}

The light-dependent and -repressed genes identified in this study $\left(^{* *}\right)$ were compared with flg22 rapidly responsive genes identified by Lyons et al. (2013) $\left(^{*}\right)$. P-value $\left({ }^{* * *}\right)$ was calculated using the hypergeometric probability formula. 
Table 2 | Top 25 genes induced and repressed by flg22 in $30 \mathrm{~min}$.

\begin{tabular}{|c|c|c|c|}
\hline AGI code & Description & flg22-up* fold change & Light-up ${ }^{* *}$ fold change \\
\hline AT5G24110 & WRKY30;_transcription_factor & 604.357 & 4.08 \\
\hline AT3G02840 & immediate_early_fungal_elicitor_family_protein & 220.241 & - \\
\hline AT4G14450 & Identical_to_Uncharacterized_protein_At4g14450 & 206.208 & - \\
\hline AT1G22810 & AP2_domain-containing_transcription_factor,_putative & 179.432 & - \\
\hline AT2G31345 & similar_to_unknown_protein_(TAIR:AT1G06135.1) & 164.362 & - \\
\hline AT3G12910 & transcription_factor & 153.511 & 3.08 \\
\hline AT1G06137 & similar_to_unknown_protein_(TAIR:AT1G06135.1) & 150.495 & - \\
\hline AT2G37430 & zinc_finger_(C2H2_type)_family_protein_(ZAT11) & 149.453 & 3.22 \\
\hline AT1G72520 & lipoxygenase,_putative & 134.598 & 2.79 \\
\hline AT5G64905 & PROPEP3_(Elicitor_peptide_3_precursor) & 96.240 & 2.2 \\
\hline AT1G56250 & ATPP2-BI4_(Phloem_protein_2-BI4) & 93.958 & 4.98 \\
\hline AT4G34410 & AP2_domain-containing_transcription_factor,_putative & 93.865 & - \\
\hline AT4G31950 & CYP82C3_(cytochrome_P450) & 93.089 & 6.73 \\
\hline AT5G05300 & similar_to_unknown_protein_(TAIR:AT3G10930.1) & 85.834 & 2.16 \\
\hline AT4G11470 & protein_kinase_family_protein & 83.563 & 3.75 \\
\hline AT4G11070 & WRKY41;_transcription_factor & 80.292 & - \\
\hline AT4G19520 & disease_resistance_protein_(TIR-NBS-LRR_class) & 80.044 & 2.16 \\
\hline AT5G42380 & CML37/CML39;_calcium_ion_binding & 71.672 & 3.03 \\
\hline \multicolumn{4}{|c|}{ flg22-REPRESSED GENES WITHIN 30 MIN } \\
\hline AT3G42550 & aspartyl_protease_family_protein & 0.009 & - \\
\hline AT3G42060 & myosin_heavy_chain-related & 0.031 & - \\
\hline AT1G66855 & similar_to_glycosyl_hydrolase_family_protein_17 & 0.032 & - \\
\hline AT4G05071 & unknown_protein & 0.035 & - \\
\hline AT4G28170 & similar_to_unknown_protein (TAIR:AT1G11120.1) & 0.036 & - \\
\hline AT1G54775 & Encodes_a_Plant_thionin_family_protein & 0.040 & - \\
\hline AT5G33390 & glycine-rich_protein & 0.044 & - \\
\hline AT5G42957 & similar_to_unknown_protein (TAIR:AT5G42955.1) & 0.047 & - \\
\hline AT1G27990 & similar_to_unknown_protein (TAIR:AT5G52420.1) & 0.050 & - \\
\hline AT3G44755 & similar_to_unknown_protein (TAIR:AT3G46360.1) & 0.051 & - \\
\hline AT4G29200 & beta_galactosidase & 0.052 & - \\
\hline AT2G15590 & similar_to_unknown_protein (TAIR:AT4G33985.1) & 0.056 & - \\
\hline AT5G28560 & unknown_protein & 0.060 & - \\
\hline AT4G27415 & unknown_protein & 0.061 & - \\
\hline AT1G34280 & unknown_protein & 0.063 & - \\
\hline AT3G59460 & similar_to_F-box_family_protein_(TAIR:AT3G6004) & 0.068 & - \\
\hline AT1G70944 & unknown_protein & 0.069 & - \\
\hline AT2G36190 & ATCWINV4 & 0.070 & - \\
\hline
\end{tabular}

*flg22-induced (upper) and -repressed (lower) genes identified by microarray analysis by Lyons et al. (2013).

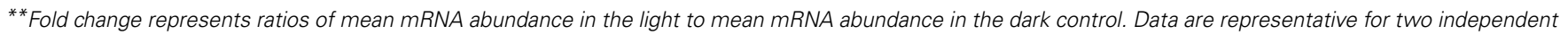
experiments $(P<0.01)$.

"-" indicates that genes were not induced nor repressed more than two times by light. 
with electron transport or energy, structural molecule activity, and chloroplasts; transcription factor TF activity genes were significantly overrepresented among the flg22-repressed lightrepressed genes.

Table 2 shows that 16 of 25 genes (64\%) that were markedly induced by flg22 after $30 \mathrm{~min}$ were also dependent on light, suggesting that light plays a critical role in the strong induction of flg22-induced genes. Thus, we further analyzed genes that are strongly $(>4.0)$ induced by flg22. We identified 889 flg22-induced $(>4.0)$ and 452 flg22-repressed $(<0.25)$ genes after 30 -min treatment with flg22. A comparison of these genes with the light-regulated genes identified in this study revealed that markedly flg22-induced genes include a larger number of lightdependent genes. Approximately $30 \%$ of the strongly induced genes (264 genes) were up-regulated in the light, but only $4.9 \%$ of those genes ( 51 genes) were down-regulated by light (Figure 1A), indicating that light plays a more significant role in the expression of genes that are largely induced by flg 22 .

CAS is a thylakoid membrane-localized $\mathrm{Ca}^{2+}$-binding protein (Han et al., 2003; Nomura et al., 2008; Vainonen et al., 2008; Weinl et al., 2008). We previously demonstrated that CAS mediates retrograde chloroplast signals to regulate flg22-induced nuclear

\section{A \\ flg22 -induced genes}

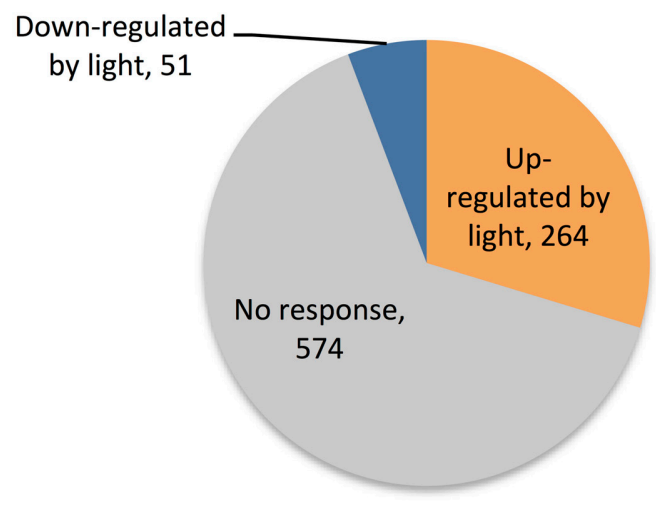

B

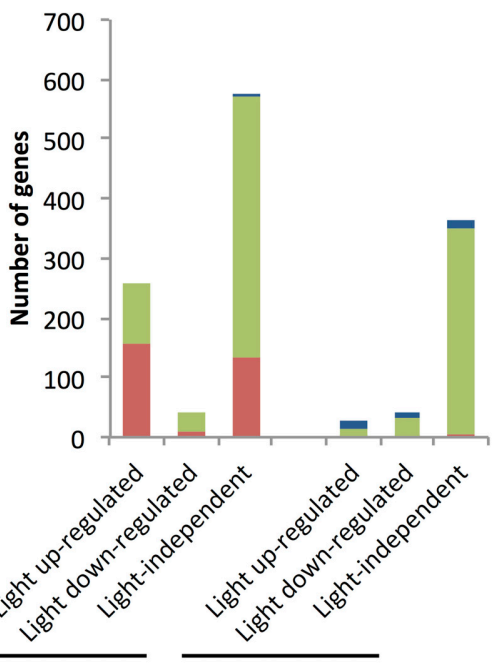

Flg22-induced genes Flg22-repressed genes

FIGURE 1 | Microarray analysis of genes up- and down-regulated by light in flg22-treated seedlings. (A) The number of genes up- and

down-regulated by light among sets of genes previously identified as strongly up- $(>4.0)$ or down-regulated $(<0.25)$ by flg22 within 30 min (Lyons et al.,

2013). (B) The number of genes previously shown to be CAS-dependent (red) or CAS-suppressed (blue) or CAS-independent (green) (Nomura et al., 2012)

\section{flg22-repressed genes}

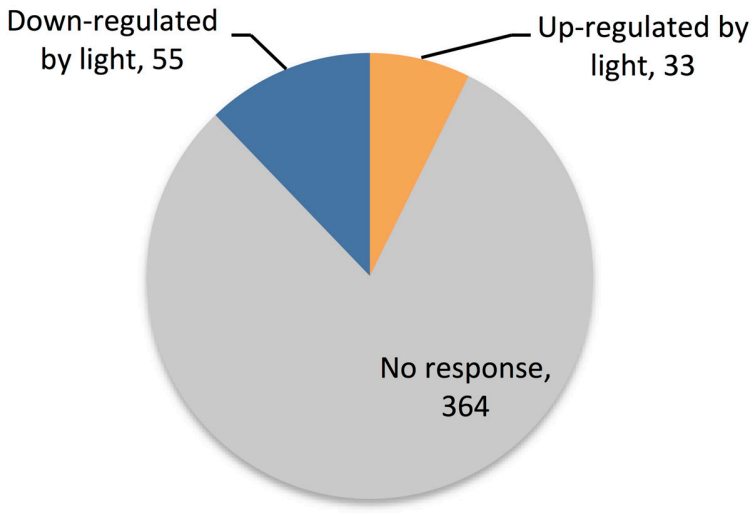

C

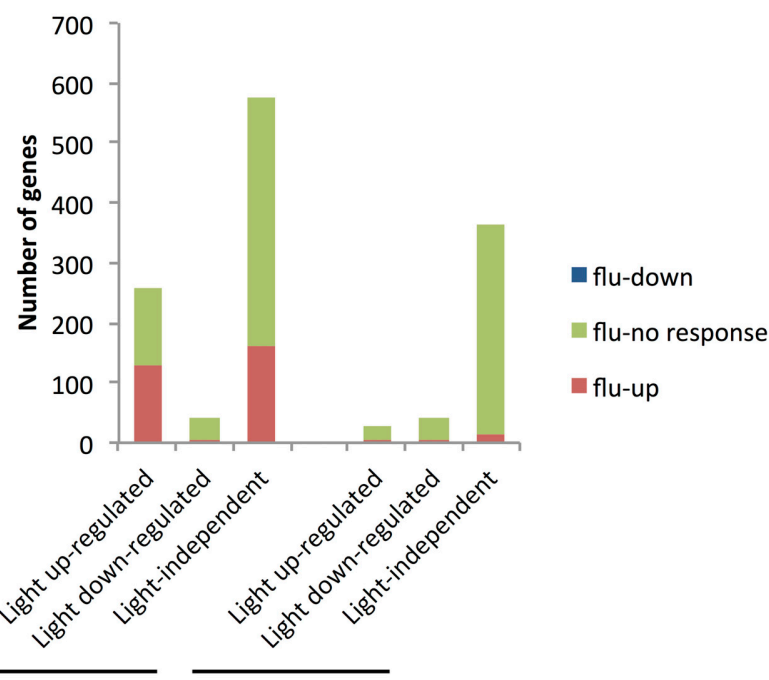

Flg22-induced genes Flg22-repressed genes

among genes that are up- (left) and down-regulated (right) by flg22 treatment (C) The number of genes previously shown to be induced in illuminated flu mutants (red) or suppressed (blue) (Laloi et al., 2007) among genes that are up- (left) and down-regulated (right) by flg22 treatment. Microarray analysis was performed with seedlings treated with flg22 for 30 min under light or dark conditions. 
gene expression. We identified 1235 genes that are induced to a lesser extent by flg22 in CAS knockout cas-1 mutants than in WT plants (CAS-dependent genes), as well as 687 genes up-regulated in cas-1 plants (CAS-repressed genes). Here, we found that the CAS-dependent genes also overlapped significantly with the flg22-induced light-dependent genes. The rapidly flg22induced light-dependent genes include 60.5\% CAS-dependent genes, whereas the flg22-induced light-independent genes contain only $23.3 \%$ CAS-dependent genes (Figure 1B). On the other hand, the rapidly flg22-induced light down-regulated genes include only one CAS-suppressed gene (Figure 1B). In contrast, the flg22-repressed genes include very few CAS-dependent genes, irrespective of light treatment. These results suggest that chloroplasts and, specifically, CAS are involved in the light-mediated control of flg22-induced nuclear defense gene expression. It has been suggested that CAS is involved in ${ }^{1} \mathrm{O}_{2}$ mediated retrograde signaling, facilitating chloroplast-mediated transcriptional reprogramming during plant immune responses. As shown in Figure 1C, 127 of 258 rapidly flg22-induced light-dependent genes overlapped significantly with $1565{ }^{1} \mathrm{O}_{2}$-responsive genes induced in illuminated $\mathrm{flu}$ mutants (fold change $>3.0$ ) (Laloi et al., 2007), suggesting that ${ }^{1} \mathrm{O}_{2}$ signaling plays a role in the light-dependent activation of flg22-induced genes.

\section{Cis-ELEMENT SEQUENCES OVERREPRESENTED IN THE LIGHT-DEPENDENT AND -INDEPENDENT flg22-INDUCED GENES}

We searched for overrepresented 6-bp motifs within the 500bp upstream region of the predicted translation start sites of the flg22-induced $(>2.0)$ and -repressed $(<0.5)$ genes using a motif discovery tool (RSAT, http://www.rsat.eu/). Several short sequences were significantly overrepresented in promoter regions of rapidly flg22-induced and -repressed genes (Tables S3, S4). The alignment of hexamers identified two known consensus sequences overrepresented in the promoters of genes rapidly induced by flg22 treatment. The most highly represented motifs in the promoters (500-bp upstream regions) of the light-independent ( $P$-value, 2e-102) and lightrepressed $(P$-value, 3.5e-23) flg22-induced genes were GGCCCA (Figure 2, sTable 3), which are part of the TCP TF-binding motifs (GGNCCCAC or GGNCCC) (Li et al., 2005). The TCPbinding motif sequence GGCCCA is also overrepresented in the light-dependent genes, suggesting that TCP is unlikely to be involved in the light-mediated response of flg22-induced genes. Furthermore, GGCCCA sequences were frequently present in the promoters of the flg22-repressed genes irrespective of light treatment.

The W-box motif (TTGACC/T) was significantly overrepresented in the promoters of the flg22-induced light-dependent genes (agtcaa; $P$-value, $8.4 \mathrm{e}-39$, gtcaac; $P$-value, $6.4 \mathrm{e}-31$ ). In contrast, the W-box was less represented in flg22-induced genes repressed by light (Figure 2). The W-box is the binding motif for WRKY family TFs, which regulate biotic and abiotic stress responses (Rushton et al., 2010). Furthermore, the W-box was not represented in the promoters of flg22repressed genes irrespective of light treatment. On the other hand, no well-known light-responsive cis-elements, such as GTelements (GR(T/A)AA(T/A)), G-box elements (CACGTG), or
I-box elements (GATAA), were overrepresented in the lightdependent flg22-induced genes. These results suggest that WRKY TFs are involved in the rapid light-dependent expression of flg22induced defense genes.

\section{qRT-PCR OF LIGHT-DEPENDENT EXPRESSION OF flg22-INDUCED GENES}

SA is a key signaling molecule in plant immune responses. We found that the expression of several key genes responsible for SA accumulation were up-regulated by light in the presence of flg22. Thus, we selected four genes involved in SA biosynthesis (EDS1, EDS5, ICS1, and PAL1) and seven TF genes that act mainly in the defense response network (ANAC042, CBP60g, WRKY6, 7, 22, 33 , and 46) as targets for quantitative reverse transcription-PCR (qRT-PCR). According to the microarray data, the flg22-induced expression of the four SA biosynthesis genes $(E D S 1, \times 2.1$; EDS5, $\times 5.79 ;$ ICS1 $\times 3.61 ;$ PAL1 $\times 12.33)$ and two of the TF genes $($ WRKY46, $\times 3.37$; ANAC042, $\times 2.57)$ was dependent on light, whereas that of the other five TF genes was not. In order to examine the effects of light on the expression of genes that are induced slowly in response to flg22 treatment, we added plants treated with flg 22 for $2 \mathrm{~h}$. As shown in Figure 3, the expression of all selected genes was up-regulated by flg22 either transiently (EDS1, ANAC042, CBP60g, WRKY22, 33, and 46) or gradually (EDS5, ICS1, PAL1, WRKY6, and 7). As expected, qRT-PCR analysis revealed that the expression of EDS1 $(\times 2.84), \operatorname{ICS} 1(\times 7.78)$, PAL1 ( $\times 10.67)$, ANAC042 $(\times 2.41)$, and WRKY46 $(\times 9.33)$ was largely dependent on light in the Arabidopsis plants treated with flg22 for $30 \mathrm{~min}$. These data are consistent with the microarray data. Although expression of EDS5 was slightly dependent on light based on qRT-PCR analysis $(\times 1.61)$, there was a significant difference between light- and dark-treated samples in microarray analysis. On the other hand, flg22-induced expression of CBP60g, WRKY7, and 22 was reduced by half in the dark compared to the light in qRT-PCR experiments $(\times 2.08, \times 2.59$, and $\times 1.77$, respectively), whereas their expression was not significantly upregulated by light in the microarray data. Expression of genes that are induced gradually by flg22, such as EDS5, ICS1, PAL1, and $W R K Y 7$, was more significantly dependent on light after $2 \mathrm{~h}$. Furthermore, it should be noted that EDS1, EDS5, ICS1, and $P A L 1$, which are involved in SA biosynthesis, showed reduced expression in the dark even before flg22 treatment, whereas the TF-related genes did not. In contrast, the expression levels of two other genes (WRKY6 and 33) were not markedly decreased in the dark, confirming the microarray data.

\section{EFFECT OF ELECTRON TRANSPORT INHIBITORS ON Flg22-INDUCED GENE EXPRESSION}

In order to examine the involvement of photosynthesis in the light-dependent defense gene expression, we analyzed the expression of flg22-induced defense genes in the presence of two specific inhibitors of photosynthetic electron transport. DCMU and DBMIB inhibit electron transport from the PS II complex to the PQ pool and from the PQ pool to the cytochrome $b_{6} / f$ complex, respectively (Trebst, 2007). Arabidopsis seedlings were pretreated with these inhibitors for $30 \mathrm{~min}$ before flg22 treatment. Electron transport rates measured using a PAM chlorophyll fluorometer were suppressed by both inhibitors to less than 
flg22-induced light-dependent genes

$\begin{array}{lll}\text { Alignment } & \text { Rev. Cpl } & \text { Score } \\ \text { A (W-box) } & \\ \text { aaggtc... . ...gacttt } & 4.76 \\ \text {. aagtca.. . . tgactt. } & 13.83 \\ \text {..agtcaa. .ttgact.. } & 34.76 \\ \text {...gtcaac } & \text { gttgac... } & 26.87 \\ \text { aaggtcaac gttgacttt } & 34.76\end{array}$

B

aagaag......ctctt

19.57

.agaaga....tcttct.

23.59

..gaagaa. .ttcttc..

33.24

...aagaaa tttctt...

7.05

aagaagaaa tttcttctt

33.24

c (TCP-motif)

$\begin{array}{ll}\text { aggccc.. } . \text {.gggcct } & 7.33 \\ \text {.ggccca. .tgggcc. } & 23.07 \\ \text {..gcccat atgggc.. } & 7.82 \\ \text { aggcccat atgggcct } & 23.07\end{array}$

D

atatat. .atatat

7.70

- tatata tatata.

atatata tatatat

E

gagaga. .tctctc

16.96

- agagag ctctct.

gagagag ctctctc flg22-induced light-repressed genes

$\begin{array}{ll}\text { Alignment Rev. Cpl } & \text { Score } \\ \text { F (TCP-motif) } & \\ \text { aggccc.. ..gggcct } & 10.05 \\ \text {.ggccca. .tgggcc. } & 19.14 \\ \text {..gcccat atgggc.. } & 11.33 \\ \text { aggcccat atgggcct } & 19.14\end{array}$

G

gccacg.......... 2.24

.ccacgt......acgtgg. 5.90

..cacgtg....cacgtg.. 11.30

...acgtgg. .ccacgt... 5.90

....cgtggc gccacg.... 2.24

gccacgtggc gccacgtggc 11.30

H

$\begin{array}{ll}\text { aggaag.......cttctt } & 4.23 \\ \text {.agaaga.. ..tcttct. } & 9.23 \\ \text {..gaagaa. .ttcttc.. } & 8.33 \\ \text {...aagaaa tttctt... } & 2.84 \\ \text { aagaagaaa tttcttctt } & 9.23\end{array}$

I

taatta taatta 8.54

taatta taatta 8.54

\section{J (TCP-motif)}

aagccc...gggctt $\quad 1.79$

.agccca. .tgggct. 7.29

..gcccat atgggc.. 11.33

aagcccat atgggctt $\quad 11.33$

the most common base pair in that position, and shown by blue characters. W-box was significantly overrepresented in the promoters of light-dependent genes, whereas TCP-motif was overrepresented in the promoters of both light-dependent and -independent genes. The score is calculated by the oligo analysis tool by default and is equivalent to $\log 10$ of the $\mathrm{E}$-value.
$10 \%$ of those in non-treated plants within $30 \mathrm{~min}$ (sFigure 2). Importantly, the light-dependent expression of all nine flg22induced genes examined was significantly suppressed by DBMIB (Figure 3) to levels similar to those observed in the dark after $30 \mathrm{~min}$ or $2 \mathrm{~h}$ of flg 22 treatment. However, flg22-induced gene expression was not significantly suppressed or was only partially suppressed by DCMU. In contrast, WRKY6 and 33, which exhibited light-independent expression, were not significantly suppressed by DBMIB or DCMU, indicating that electron transport inhibitors do not affect the light-independent expression of flg22-induced defense genes. These results suggest that the photosynthetic electron flow plays a key role in regulating the light-dependent expression of flg22-induced defense genes.

\section{EFFECTS OF LIGHT ON flg22-INDUCED SA ACCUMULATION}

We found that the flg22-induced expression of a set of genes involved in SA biosynthesis (EDS1, ICS1, EDS5, and PAL1) and regulation (CBP60g, WRKY7, and 46; Kim et al., 2006; Zhang et al., 2010; van Verk et al., 2011) was dependent on light (Figure 2). Therefore, to elucidate the role of light in flg22induced SA biosynthesis, we investigated free SA accumulation in the leaves of Arabidopsis seedlings treated with flg22 under 

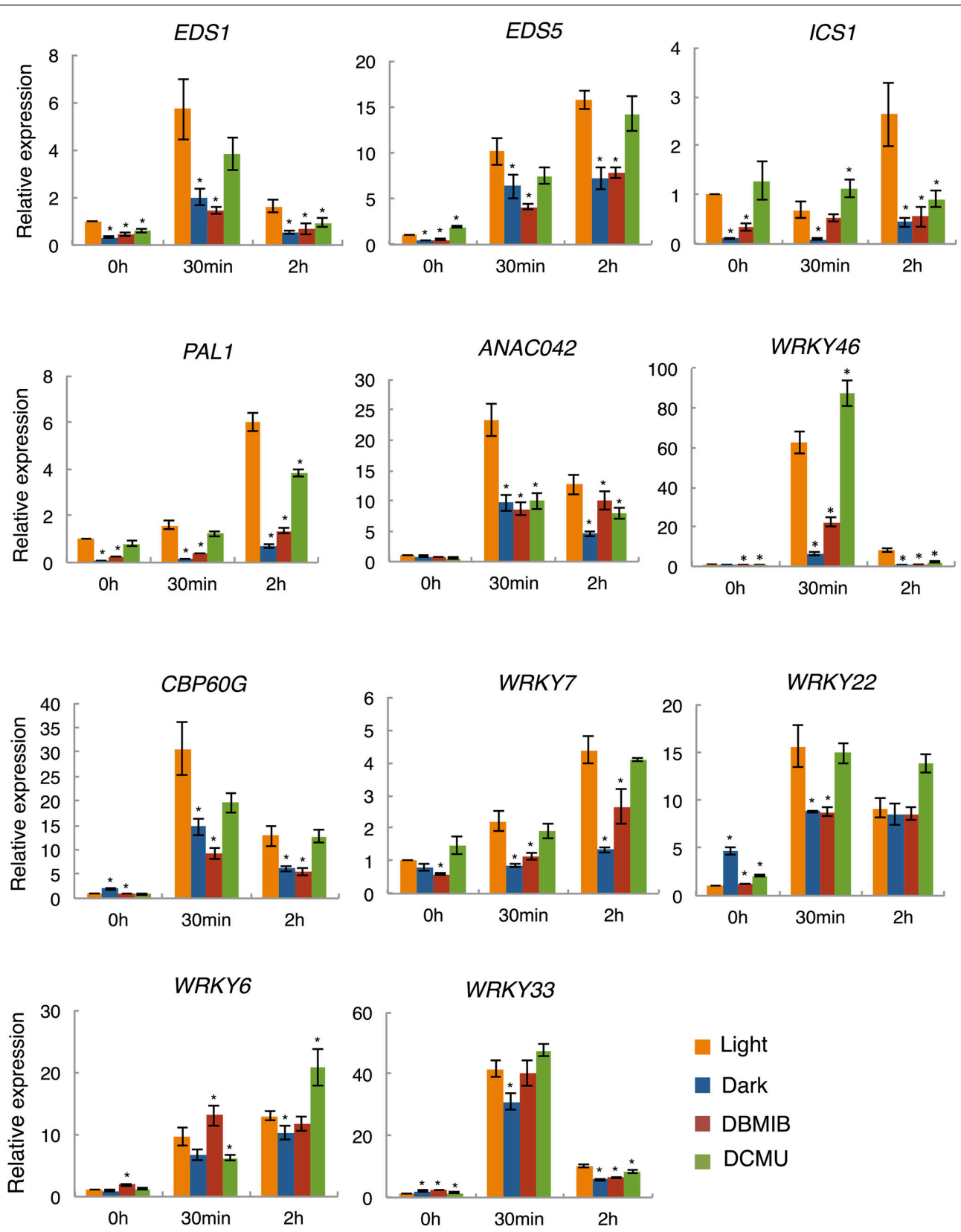

FIGURE 3 | qRT-PCR analysis of flg22-induced gene expression in the dark and light, and in the presence of photosynthesis inhibitors.

Plants were treated with $1 \mu \mathrm{M}$ flg22 in the light (orange) or dark (blue) for $30 \mathrm{~min}$ and $2 \mathrm{~h}$, respectively. As indicated, plants were also pretreated with DBMIB $(5 \mu \mathrm{M}$; red) or DCMU $(8 \mu \mathrm{M}$; green) for $30 \mathrm{~min}$ before flg22 treatment. UBQ10 was used as an internal standard. Results shown are mean + s.e.m. from triplicate technical replicates from one of three representative experiments with similar results. $P$-values for qRT-PCR data were calculated using $t$-tests and are indicated by $* p<0.005$. light and dark conditions (Figure 4). Upon flg22 treatment, Arabidopsis accumulated less SA in the dark than in the light. These results indicate that light is involved in the activation of SA biosynthesis by flg22.

\section{DISCUSSION}

It has been suggested that light is necessary for robust and precise immune responses in plants (Roden and Ingle, 2009; Wang et al., 2011). Several reports have suggested the involvement of photoreceptors, such as phytochromes (Griebel and Zeier, 2008) and cryptochromes (Jeong et al., 2010; Wu and Yang, 2010), in the plant immune system. It has also been indicated that photosynthesis is involved in the activation of SA biosynthesis, PR1 gene expression, and HR against infection by pathogens (Jelenska et al., 2007; Kangasjärvi et al., 2012; Kim et al., 2012). We previously demonstrated that the chloroplast $\mathrm{Ca}^{2+}$-binding protein CAS plays a role in flg22-induced immune responses through retrograde signals originating in chloroplasts to regulate 


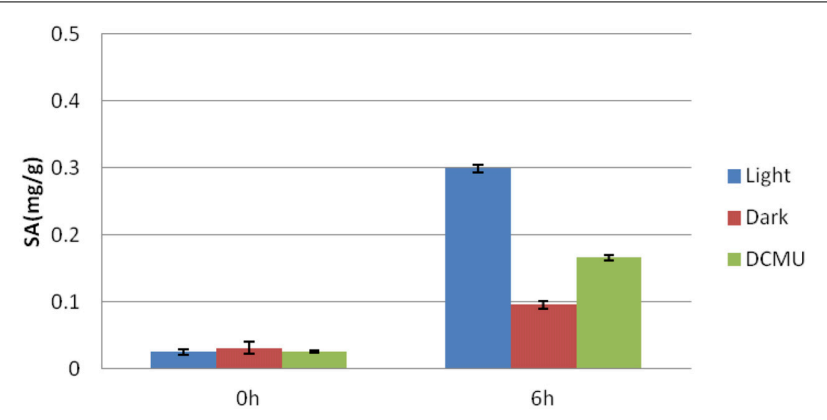

FIGURE 4 | Light-dependent salicylic acid (SA) accumulation.

Flg22-induced SA accumulation was measured in leaves in the light or dark. As indicated, some plants were treated with DCMU $(8 \mu \mathrm{M})$ for 30 min before flg22 treatment. Bars indicate the standard error of the mean (s.e.m.).

defense gene expression in the nucleus (Nomura et al., 2012). To learn more about the roles of chloroplasts and photosynthesis in PAMP-induced immunity, we investigated the effects of light and photosynthesis inhibitors on bacterial flagellin peptide flg22-induced expression of nuclear-encoded defense genes in Arabidopsis.

Microarray analysis revealed that a number of the rapidly flg22-induced genes are dependent on light, but not the flg22repressed genes. These results suggest that light is required for the activation of gene expression induced by flg22. Furthermore, we examined the effects of photosynthesis inhibitors on flg22induced gene expression in the light. The flg22-induced expression of all light-induced genes examined (EDS1, EDS5, ICS1, ANAC042, PAL1, CBP60g, WRKY7, 22, and 46) was significantly suppressed by DBMIB, and the expression of ICS1 and ANAC042 was partially suppressed by DCMU. However, these photosynthesis inhibitors did not affect flg22-induced expression of light-independent genes (WRKY6 and 33). These results suggest that photosynthesis mediates the light-dependent expression of flg22-induced genes. DCMU and DBMIB inhibit the flow of electrons between PSII and the PQ pool, and between the PQ pool and PSI, respectively. In fact, both inhibitors significantly reduced the electron transport activity between PSII and PSI (sFigure 3), suggesting that the intersystem electron flow between PSII and PSI may be involved in the light-dependent regulation of flg22-induced gene expression.

It should be noted that DBMIB showed more significant effects on light-dependent gene expression induced by flg22 than DCMU. DBMIB reduced the expression of all eight lightdependent defense genes examined to the levels found in darkadapted plants, while DCMU treatment did not significantly change the expression of defense genes (by more than twofold) except for two genes, ANACO42 and ICS1. DCMU and DBMIB have opposite effects on the redox state of the PQ pool: the PQ pool is oxidized by DCMU treatment and reduced by DBMIB treatment. Thus, the redox state of the PQ pool may be important as a signal for light-dependent flg22-induced defense gene expression in plant immunity. These findings suggest that modification of the redox state plays a critical role in the generation of chloroplast-derived signals to control nuclearencoded defense and stress-responsive genes. On the other hand, it is known that DBMIB inhibits not only photosynthetic electron transport in chloroplasts, but also mitochondrial electron transport. Although we used a low concentration of DBMIB, it is possible that it partially affects mitochondrial functions.

Previous reports demonstrated that reduction of the PQ pool by DBMIB and excess light promoted the expression of immune genes. Furthermore, recent transcription analysis of DBMIBtreated plants revealed that PQ pool reduction promoted the expression of 798 stress-responsive genes (Jung et al., 2013). Thus, it has been suggested that the PQ redox state triggers the induction of immunity- and stress-related genes. In accordance with these findings, DBMIB-induced genes were significantly overrepresented among the flg22-induced light-dependent genes $(>4.0)(28.0 \%)$, but less in the group of flg22-induced genes repressed by light and light-independent genes $(16.0 \%)$ (sFigure 3 ). These results suggest that PQ-pool redox signaling is also involved in the light-dependent expression of flg22-induced genes. However, the light-dependent expression of flg22-induced genes was largely suppressed by DBMIB (Figure 3). A mechanism linking the flg22-induced signaling and PQ-pool redox signaling remains elusive. Further studies are needed to explore the discrepancy regarding the effects of DBMIB effects on immunity-related gene expression in the presence and absence of flg22, which may shed lights on the role of PQ-pool redox in retrograde signaling to control nuclear-encoded immunity- and stress-related genes.

Perturbation of photosynthetic electron flow promotes the generation of ROS, including ${ }^{1} \mathrm{O}_{2}$ in PS II, and $\mathrm{O}_{2}^{-}$and $\mathrm{H}_{2} \mathrm{O}_{2}$ in PS I. ${ }^{1} \mathrm{O}_{2}$ and $\mathrm{H}_{2} \mathrm{O}_{2}$ may be involved in the retrograde signals to activate nuclear-encoded defense gene expression (Kangasjärvi et al., 2013) and the HR (Kim et al., 2012). Thus, ROS are candidate retrograde signals that mediate the photosynthesis-dependent regulation of nuclear-encoded defense genes. Photosynthesis inhibitors and dark conditions may suppress the electron flow-dependent ROS immune signals and subsequent defense gene expression. It is suggested that PAMPs somehow perturb the photosynthetic electron flow that leads to the generation of chloroplast-derived ROS immune signals. Previously, we demonstrated that PAMP signals are rapidly transmitted to chloroplasts to generate a transient increase in $\mathrm{Ca}^{2+}$ in chloroplasts (Nomura et al., 2012). Furthermore, our previous work implicated CAS in the generation of ${ }^{1} \mathrm{O}_{2}$-mediated signals to activate the flg22-induced expression of several defense genes. Recently, it was also reported that PAMPs cause a rapid decrease in NPQ after 30 min (Manzoor et al., 2012). Thus, chloroplasts may be able to quickly recognize PAMP signals, leading to changes in photosynthesis.

SA biosynthesis induced by pathogenic infection is dependent on light (Zeier et al., 2004); consistent with this, we showed that flg22-induced accumulation of SA is also dependent on light. SA biosynthesis in plants involves two distinct pathways: the ICS and the PAL pathways. Both pathways originate from chorismate, which is the end-product of the shikimate pathway (Dempsey et al., 2011). We found that light-induced genes activated by flg22 
include a number of genes involved in SA biosynthesis, including EDS1, PAD4, SAG101, EDS5, PAL1, and PAL2. qRT-PCR analysis revealed that the flg22-induced expression of EDS1, ICS1, EDS5, and PAL1 is suppressed by DBMIB. Light is also required for the flg22-induced expression of two TFs, CBP60g (Zhang et al., 2010; Wang et al., 2011) and WRKY46 (van Verk et al., 2011), which are involved in the activation of SA biosynthesis genes. Furthermore, it should be noted that light is responsible for the expression of genes involved in SA biosynthesis even prior to flg22-treatment. Light may be involved in the priming of SA biosynthesis genes. It is known that SA accumulation is elevated in the light (Mateo et al., 2006). Thus, flg22-induced SA accumulation in the light may be partially due to the direct photosynthesis-mediated activation of SA accumulation in chloroplasts. Taken together, these results suggest that light activates the expression of SA biosynthesis genes through photosynthesis-mediated immune signals, leading to SA accumulation.

We found that W-box sequences are significantly enriched in the promoters of the light-dependent flg22-induced genes. The W-box is the binding motif for WRKY family TFs (Rushton et al., 2010). The expression of more than 70\% of WRKY gene family members in Arabidopsis is responsive to pathogenic infection and SA treatment (Dong et al., 2003). These findings suggest that WRKY TFs play an important role in the transcription of flg22-induced defense genes in the light. Interestingly, the light-dependent flg22-induced genes ( $>4.0$ ) include just three WRKY TF genes (WRKY30, 46, and 53), while 12 WRKY TF genes (WRKY6, 11, 22, 26, 33, 40, 41, 55, 62, and 70) were identified among the light-independent or -repressed flg22-induced genes. Further analyses of these three WRKY TFs may shed light on the photosynthesis-mediated immune signals.

Contrastingly, the TCP-binding motif sequences are significantly overrepresented in the promoters of most flg22-regulated genes, except for light- and flg22-repressed genes. TCP family TFs are involved in the transcriptional regulation of genes controlling the cell cycle, growth, development, circadian clock, and jasmonic acid biosynthesis (Trémousaygue et al., 2003; Li et al., 2005; Welchen and Gonzalez, 2005, 2006; Schommer et al., 2008; Hervé et al., 2009; Pruneda-Paz et al., 2009). TCP TFs may also be involved in $\mathrm{Ca}^{2+}$-dependent transcriptional regulation in Arabidopsis (Whalley et al., 2011). Furthermore, the GGCCCA and AGCCCA motifs are also similar to a FORC ${ }^{A}$ promoter element (T/ATGGGC) (Evrard et al., 2009). FORC ${ }^{A}$-mediated promoter activity is induced by SA under constant light exposure, whereas SA does not activate the $\mathrm{FORC}^{\mathrm{A}}$-mediated promoter under constant darkness (Evrard et al., 2009). The further characterization of TCP TFs and FORC ${ }^{\mathrm{A}}$ promoter elements may provide insight into the light-mediated control of flg22-repressed genes.

In summary, this study revealed that both the up- and downregulation of defense-related genes by flg22 is dependent on light to a large extent, and suggested that photosynthesis plays a role in the light-dependent regulation of flg22-responsive genes. It is also suggested that chloroplasts produce lightdependent retrograde signals to regulate flg22-induced nuclear gene expression. Alternatively, photosynthesis may indirectly influence defense responses. Our findings further suggest that ROS and the redox state of the PQ pool are involved in this lightdependent chloroplast-mediated immune signaling. However, the molecular mechanisms linking photosynthesis and defense gene expression remain largely elusive. Further experiments are needed to clarify light-dependent retrograde chloroplast-to-nucleus signaling, which optimizes pathogen-induced defense responses in a fluctuating light environment.

\section{ACKNOWLEDGMENT}

We thank Y. Ishizaki for helpful discussion. We acknowledge Y. Yamamoto (KIST BIC) for technical assistance with the LC/MS/MS. This work was supported by JSPS and MEXT Grantsin-Aid for Scientific Research to Takashi Shiina (24657036, 25291065, 25120723) and Hironari Nomura (25870423), a grant from the Mitsubishi Foundation to Takashi Shiina, and a grant for high-priority study from Kyoto Prefectural University. This work was also supported by JSPS-DGHE International grant between Japan and Indonesia.

\section{SUPPLEMENTARY MATERIAL}

The Supplementary Material for this article can be found online at: http://www.frontiersin.org/journal/10.3389/fpls.2014. 00531/abstract

\section{REFERENCES}

Cerrudo, I., Keller, M. M., Cargnel, M. D., Demkura, P. V., de Wit, M., Patitucci, M. S., et al. (2012). Low red/far-red ratios reduce Arabidopsis resistance to Botrytis cinerea and jasmonate responses via a COI1- JAZ10-dependent, salicylic acid-independent mechanism. Plant Physiol. 158, 2042-2052. doi: 10.1104/pp.112.193359

Chisholm, S. T., Coaker, G., Day, B., and Staskawicz, B. J. (2006). Host-microbe interactions: shaping the evolution of the plant immune response. Cell 124, 803-814. doi: 10.1016/j.cell.2006.02.008

Dempsey, D. A., Volt, A. C., Wildermuth, M. C., and Klessig, D. F. (2011). 'Salicylic acid biosynthesis and metabolism.' Arabidopsis Book 9:e0156. doi: 10.1199/tab.0156

Dong, J., Chen, C., and Chen, Z. (2003). Expression profiles of the Arabidopsis WRKY gene superfamily during plant defense response. Plant Mol. Biol. 51, 21-37. doi: 10.1023/A:1020780022549

Evrard, A., Ndatimana, T., and Eulgem, T. (2009). FORC ${ }^{A}$, a promoter element that responds to crosstalk between defense and light signaling. BMC Plant Biol. 9:2. doi: 10.1186/1471-2229-9-2

Fragnière, C., Serrano, M., Abou-Mansour, E., Métraux, J. P., and L'Haridon, F. (2011). Salicylic acid and its location in response to biotic and abiotic stress. FEBS Lett. 585, 1847-1852. doi: 10.1016/j.febslet.2011.04.039

Fujita, M., Fujita, Y., Noutoshi, Y., Takahashi, F., Narusaka, Y., YamaguchiShinozaki, K., et al. (2006). Crosstalk between abiotic and biotic stress responses: a current view from the points of convergence in the stress signaling networks. Curr. Opin. Plant Biol. 9, 1-7. doi: 10.1016/j.pbi.2006. 05.014

Göhre, V., Jones, A. M., Sklenáø, J., Robatzek, S., and Weber, A. P. (2012). Molecular crosstalk between PAMP-triggered immunity and photosynthesis. Mol. Plant Microb. Interact. 25, 1083-1092. doi: 10.1094/MPMI-1111-0301

Göhre, V., and Robatzek, S. (2008). Breaking the barriers: microbial effector molecules subvert plant immunity. Annu. Rev. Phytopathol. 46, 189-215. doi: 10.1146/annurev.phyto.46.120407.110050

Griebel, T., and Zeier, J. (2008). Light regulation and daytime dependency of inducible plant defenses in Arabidopsis: phytochrome signaling controls systemic acquired resistance rather than local defense. Plant Physiol. 147, 790-801. doi: 10.1104/pp.108.119503 
Han, S., Tang, R., Anderson, L. K., Woerner, T. E., and Pei, Z. M. (2003). A cell surface receptor mediates extracellular $\mathrm{Ca}(2+)$ sensing in guard cells. Nature 425, 196-200. doi: 10.1038/nature01932

Hervé, C., Dabos, P., Bardet, C., Jauneau, A., Auriac, M. C., Ramboer, A., et al. (2009). In vivo interference with AtTCP20 function induces severe plant growth alterations and deregulates the expression of many genes important for development. Plant Physiol. 149, 1462-1477. doi: 10.1104/pp.108. 126136

Hua, J. (2013). Modulation of plant immunity by light, circadian rhythm and temperature. Curr. Opin. Plant Biol. 16, 1-8. doi: 10.1016/j.pbi.2013.06.017

Ishihama, N., Yamada, R., Yoshioka, M., Katou, S., and Yoshioka, H. (2011). Phosphorylation of the Nicotiana benthamiana WRKY8 transcription factor by MAPK functions in the defense response. Plant Cell 23, 1153-1170. doi: 10.1105/tpc.110.081794

Jelenska, J., Yao, N., Vinatzer, B. A., Wright, C. M., Brodsky, J. L., and Greenberg, J. T. (2007). A J domain virulence effector of Pseudomonas syringae remodels host chloroplasts and suppresses defenses. Curr. Biol. 17, 499-508. doi: 10.1016/j.cub.2007.02.028

Jeong, R. D., Chandra-Shekara, A. C., Barman, S. R., Navarre, D., Klessig, D. F., Kachroo, A., et al. (2010). Cryptochrome 2 and phototropin 2 regulate resistance protein-mediated viral defense by negatively regulating an E3 ubiquitin ligase. Proc. Natl. Acad. Sci. U.S.A. 107, 13538-13543. doi: 10.1073/pnas. 1004529107

Jung, H.-S., Crisp, P., Estavillo, G. M., Cole, B., Hong, F., Mockler, T. C., et al. (2013). Subset of heat-shock transcription factors required for the early response of Arabidopsis to excess light. Proc. Natl. Acad. Sci. U.S.A. 110, 14474-14479. doi: 10.1073/pnas.1311632110

Kangasjärvi, S., Neukermans, J., Li, S., Aro, E. M., and Noctor, G. (2012). Photosynthesis, photorespiration, and light signalling in defense responses. J. Exp. Bot. 63, 1619-1636. doi: 10.1093/jxb/err402

Kangasjärvi, S., Tikkanen, M., Durian, G., and Aro, E. M. (2013). Photosynthetic light reactions - an adjustable hub in basic production and plant immunity signaling. Plant Physiol. Biochem. 81, 128-134. doi: 10.1016/j.plaphy.2013. 12.004

Karpiński, S., Szechyńska-Hebda, M., Wituszyńska, W., and Burdiak, P. (2013). Light acclimation, retrograde signalling, cell death and immune defences in plants. Plant Cell Environ. 36, 736-744. doi: 10.1111/pce.12018

Kim, C., Meskauskiene, R., Zhang, S., Lee, K. P., Ashok, M. L., Blajecka, K., et al. (2012). Chloroplasts of Arabidopsis are the source and a primary target of a plant-specific programmed cell death signaling pathway. Plant Cell. 24, 3026-3039. doi: 10.1105/tpc.112.100479

Kim, K. C., Fan, B., and Chen, Z. (2006). Pathogen-induced Arabidopsis WRKY7 is a transcriptional repressor and enhances plant susceptibility to Pseudomonas syringae. Plant Physiol. 142, 1180-1192. doi: 10.1104/pp.106.082487

Laloi, C., Stachowik, M., Pers-Kamczyc, E., Warzych, E., Murgia, I., and Apel, K. (2007). Cross-talk between singlet oxygen- and hydrogen peroxide- dependent signaling of stress responses in Arabidopsis thaliana. Proc. Natl. Acad. Sci. U.S.A. 104, 672-677. doi: 10.1073/pnas.0609063103

Li, C. X., Potuschak, T., Colón-Carmona, A., Gutiérrez, R. A., and Doerner, P. (2005). Arabidopsis TCP20 links regulation of growth and cell division control pathways. Proc. Natl. Acad. Sci. U.S.A. 102. 12978-12983. doi: 10.1073/pnas.0504039102

Lyons, R., Iwase, A., Gänsewig, T., Sherstnev, A., Duc, C., Barton, G. J., et al. (2013). The RNA-binding protein FPA regulates flg22-triggered defense responses and transcription factor activity by alternative polyadenylation. Sci. Rep. 3, 2866. doi: $10.1038 /$ srep02866

Manzoor, H., Chiltz, A., Madani, S., Vatsa, P., Schoefs, B., Pugin, A., et al. (2012). Calcium signatures and signaling in cytosol and organelles of tobacco cells induced by plant defense elicitors. Cell Calcium. 51, 434-444. doi: 10.1016/j.ceca.2012.02.006

Mateo, A., Funck, D., Mühlenbock, P., Kular, B., Mullineaux, P. M., and Karpinski, S. (2006). Controlled levels of salicylic acid are required for optimal photosynthesis and redox homeostasis. J. Exp. Bot. 57, 1795-1807. doi: 10.1093/jxb/ erj196

Michael, T. P., Mockler, T. C., Breton, G., McEntee, C., Byer, A., Trout, J. D., et al. (2008). Network discovery pipeline elucidates conserved time-of-day-specific cis-regulatory modules. PLoS Genetics 4:e14. doi: 10.1371/journal.pgen.00 40014
Mühlenbock, P., Szechynska-Hebda, M., Plaszczyca, M., Baudo, M., Mateo, A., Mullineaux, P. M., et al. (2008). Chloroplast signaling and LESION SIMULATING DISEASE1 regulate crosstalk between light acclimation and immunity in Arabidopsis. Plant Cell 20, 2339-2356. doi: 10.1105/tpc.108. 059618

Nomura, H., Komori, T., Kobori, M., Nakahira, Y., and Shiina, T. (2008). Evidence for chloroplast control of external $\mathrm{Ca}^{2+}$-induced cytosolic $\mathrm{Ca}^{2+}$ transients and stomatal closure. Plant J. 53, 988-98. doi: 10.1111/j.1365-313X.2007. 03390.x

Nomura, H., Komori, T., Uemura, S., Kanda, Y., Shimotani, K., Nakai, K., et al. (2012). Chloroplast-mediated activation of plant immune signalling in Arabidopsis. Nat. Commun. 3, 926. doi: 10.1038/ncomms1926

Pruneda-Paz, J. L., Breton, G., Para, A., and Kay, S. A. (2009). A functional genomics approach reveals $\mathrm{CHE}$ as a component of the Arabidopsis circadian clock. Science 323, 1481-1485. doi: 10.1126/science.1167206

Roberts, M. R., and Paul, N. D. (2006). Seduced by the dark side: integrating molecular and ecological perspectives on the influence of light on plant defence against pests and pathogens. New Phytol. 170, 677-699. doi: 10.1111/j.14698137.2006.01707.x

Roden, L. C., and Ingle, R. A. (2009). Lights, rhythms, infection: the role of light and the circadian clock in determining the outcome of plantpathogen interactions. Plant Cell 21, 2546-2552. doi: 10.1105/tpc.109. 069922

Rushton, P. J., Somssich, I. E., Ringler, P., and Shen, Q. J. (2010). WRKY transcription factors. Trends Plant Sci. 15, 247-258. doi: 10.1016/j.tplants.2010. 02.006

Schommer, C., Palatnik, J. F., Aggarwal, P., Chételat, A., Cubas, P., Farmer, E. E., et al. (2008). Control of jasmonate biosynthesis and senescence by miR319 targets. PLoS Biol. 6:e230. doi: 10.1371/journal.pbio.0060230

Szechyńska-Hebda, M., and Karpiński, S. (2013). Light intensity-dependent retrograde signalling in higher plants. J. Plant Physiol. 170, 1501-1516. doi: 10.1016/j.jplph.2013.06.005

Trebst, A. (2007). Inhibitors in the functional dissection of the photosynthetic electron transport system. Photosynth. Res. 92, 217-224. doi: 10.1007/s11120007-9213-x

Trémousaygue, D., Garnier, L., Bardet, C., Dabos, P., Hervé, C., and Lescure, B. (2003). Internal telomeric repeats and 'TCP domain' protein-binding sites cooperate to regulate gene expression in Arabidopsis thaliana cycling cells. Plant J. 33, 957-966. doi: 10.1046/j.1365-313X.2003. 01682.x

Vainonen, J. P., Sakuragi, Y., Stael, S., Tikkanen, M., Allahverdiyeva, Y., Paakkarinen, V., et al. (2008). Light regulation of CaS, a novel phosphoprotein in the thylakoid membrane of Arabidopsis thaliana. FEBS J. 275, 1767-1777. doi: 10.1111/j.1742-4658.2008.06335.x

van Verk, M. C., Bol, J. F., and Linthorst, H. J. (2011). WRKY transcription factors involved in activation of SA biosynthesis genes. BMC Plant Biol. 11:89. doi: 10.1186/1471-2229-11-89

Wang, W., Barnaby, J. Y., Tada, Y., Li, H., Tör, M., Caldelari, D., et al. (2011). Timing of plant immune responses by a central circadian regulator. Nature 470, 110-114. doi: 10.1186/1471-2229-11-89

Weinl, S., Held, K., Schlücking, K., Steinhorst, L., Kuhlgert, S., Hippler, M., et al. (2008). A plastid protein crucial for Ca2+-regulated stomatal responses. New Phytol. 179, 675-686. doi: 10.1111/j.1469-8137.2008. 02492.x

Welchen, E., and Gonzalez, D. H. (2005). Differential expression of the Arabidopsis cytochrome $c$ genes $C y t c-1$ and $C y t c-2$. Evidence for the involvement of TCPdomain protein-binding elements in anther- and meristem-specific expression of the Cytc-1 gene. Plant Physiol. 139, 88-100. doi: 10.1104/pp.105. 065920

Welchen, E., and Gonzalez, D. H. (2006). Overrepresentation of elements recognized by TCP-domain transcription factors in the upstream regions of nuclear genes encoding components of the mitochondrial oxidative phosphorylation machinery. Plant Physiol. 141, 540-545. doi: 10.1104/pp.105. 075366

Whalley, H. J., Sargeant, A. W., Steele, J. F. C., Lacoere, T., Lamb, R., Saunders, N. J., et al. (2011). Transcriptomic analysis reveales calcium regulation of specific promoter motifs in Arabidopsis. Plant Cell 23, 4079-4095. doi: 10.1105/tpc. 111.090480 
Wu, L., and Yang, H. Q. (2010). CRYPTOCHROME 1 is implicated in promoting R protein-mediated plant resistance to Pseudomonas syringae in Arabidopsis. Mol. Plant 3, 539-548. doi: 10.1093/mp/ssp107

Zeier, J., Pink, B., Mueller, M. J., and Berger, S. (2004). Light conditions influence specific defence responses in incompatible plant-pathogen interactions: uncoupling systemic resistance from salicylic acid and PR-1 accumulation. Planta 219, 673-683. doi: 10.1007/s00425-004-1272-Z

Zhang, Y., Xu, S., Ding, P., Wang, D., Cheng, Y. T., He, J., et al. (2010). Control of salicylic acid synthesis and systemic acquired resistance by two members of a plant-specific family of transcription factors. Proc. Natl Acad. Sci. U.S.A. 107, 18220-18225. doi: 10.1073/pnas.1005225107

Conflict of Interest Statement: The authors declare that the research was conducted in the absence of any commercial or financial relationships that could be construed as a potential conflict of interest.
Received: 06 June 2014; accepted: 18 September 2014; published online: 09 October 2014.

Citation: Sano S, Aoyama M, Nakai K, Shimotani K, Yamasaki K, Sato MH, Tojo D, Suwastika IN, Nomura $H$ and Shiina $T$ (2014) Light-dependent expression of flg22-induced defense genes in Arabidopsis. Front. Plant Sci. 5:531. doi: 10.3389/fpls. 2014.00531

This article was submitted to Plant-Microbe Interaction, a section of the journal Frontiers in Plant Science.

Copyright (C) 2014 Sano, Aoyama, Nakai, Shimotani, Yamasaki, Sato, Tojo, Suwastika, Nomura and Shiina. This is an open-access article distributed under the terms of the Creative Commons Attribution License (CC BY). The use, distribution or reproduction in other forums is permitted, provided the original author(s) or licensor are credited and that the original publication in this journal is cited, in accordance with accepted academic practice. No use, distribution or reproduction is permitted which does not comply with these terms. 